\title{
Research on Motivation Theory and Enlightenment to Teaching
}

\author{
Yameng Zhang \\ Zhejiang Yuexiu University of Foreign Languages, Shaoxing, China \\ Email: 229146776@qq.com
}

How to cite this paper: Zhang, Y. M. (2021). Research on Motivation Theory and Enlightenment to Teaching. Open Journal of Social Sciences, 9, 476-483.

https://doi.org/10.4236/jss.2021.92031

Received: January 28, 2021

Accepted: February 23, 2021

Published: February 26, 2021

Copyright $\odot 2021$ by author(s) and Scientific Research Publishing Inc. This work is licensed under the Creative Commons Attribution International License (CC BY 4.0).

http://creativecommons.org/licenses/by/4.0/

\begin{abstract}
The paper integrates psychological motivation theory and analyzes its role in second language learning. The motivation of psychological exploration is conducted to a better understanding of motivation and human behavior from different perspectives. In this way, we can recognize the impact of external forces on human beings, go deep into the inner world, and examine the relationship between learners and their surrounding environment, It also attempts to explore the inspiration of motivation theory on learning second language, so that teachers can get better teaching results and pave the way for future research.
\end{abstract}

\section{Keywords}

Second Language Learning, Motivation, Psychology, Teaching

\section{Introduction}

Motivation is one of the most important factors in psychology. Most theories about motivation mainly explain people's behavior and way of thinking (Dornyei, 2001). Generally speaking, the main purpose of learning a second language is to achieve two goals. They are represented by the dichotomy of synthesis and instrumental motivation. Motivational learners learn the language so that they can use it to communicate with other people and better understand their lives. The most successful learners are those who like to speak and appreciate the target language culture, eager to be familiar with or even want to use the language in daily life. Such kinds of students are typical language learners with comprehensive motivation. They actively seize every possible opportunity in the process of acquiring the target language, cultivating their oral English, reading, writing and listening ability. However, in some learning contexts, instrumental motiva- 
tion seems to determine whether second language learners succeed or not. This is the characteristic of the second language acquisition learners, and there is little or no opportunity to use the target language to integrate into the community or interact with its members. In this case, learners passively strengthen one or more abilities of speaking, reading, writing and listening according to their actual needs. After reaching the goal, most learners with instrumental motivation may lose their short-term motivation and stop learning. Previous literature on motivation had shown that there was no clear distinction between integration oriented learners and instrument oriented learners in the process of learning a second language. Both of them may be effective factors to motivate success. The two types of motivation are not necessarily mutually exclusive. Second language learning is rarely motivated by instrumental or fully integrated attitudes. And since 1960s, with the development of cognitive psychology and social constructivism, western educators have already made a lot of researches on learner autonomy. Learner autonomy is a capacity for detachment, critical reflection, decision-making, and independent action. It presupposes, but also entails, that the learner will develop a particular kind of psychological relation to the process and content of learning. The capacity for autonomy will be displayed both in the way the learner learns and in the way they transfer what has been learned to wider contexts.

\section{Motivation Classification}

\subsection{Definition of Motivation}

There is no doubt that how to define motivation is the first step psychologists need to take before further research. Motivation has been defined as the goal of learning a second language. The psychological school explains the concept of motivation from different angles. For example, from the perspective of social constructivism, it is defined as follows: Motivation can be constructed as a state of cognitive and emotional arousal, which leads to conscious decision-making action, and it produces a period of sustained intellectual and physical effort in order to achieve a previous goal. And some components of motivations are generally accepted: 1) the goal of language learning; 2) the effort to achieve the goal; 3) the maintenance of the effort.

\subsection{Intrinsic and Extrinsic Motivation}

According to the source of learning motivation, learning motivation can be divided into two types: internal and external. Intrinsic motivation activities are activities that have no obvious return except the activities themselves. It seems that people are eager to participate in these activities for their own sake, not because they lead to external rewards, while internal behaviors are designed to bring about some internal reward consequences, namely, the feeling of ability and self-determination. It can explain why some foreign language learners maintain strong enthusiasm and motivation for a long time, as well as various other beha- 
viors that need not be strengthened. At the same time, it can also explain the various psychological processes involved in successful pursuit. For them, the learning experience itself, the ability to promote feedback and a sense of self-determination are the best rewards.

Deci also pointed out that learners with extrinsic motivation do activities in order to obtain some rewards or avoid some punishment outside the activities themselves (Deci, 1975). This motivation refers to the learning situation, in which the reason for doing tasks is whether they are interested in the task itself (or the broader learning effort). Taking on tasks may be something lean feels compelled to do, rather than something it really wants to do. It stems from the desire to receive any form of reward, such as decent TOEFL scores and financial rewards, or, in some cases, to avoid punishment. However, externally driven behavior is addictive in nature. Learners can try their every effort to achieve their goals and get the expected reward. However, when the reward is no longer useful, they will lose motivation.

Researchers in second language acquisition field strongly support intrinsic motivation. Intrinsic motivation is obviously superior to external long-term retention. In fact, it is difficult to find out which one plays a more important role in the success of second language learning. In most cases, intrinsic motivation and extrinsic motivation are effective for learners. However, it is reasonable to assume that our language learners usually have a better chance of success and inner form of realization ability and autonomy, although feedback leads to the increase of self ability and self feeling, which is an external reward and shows further intrinsic motivation (Brown, 1994).

Motivation of integrating tools is the same as internal and external structure to a certain extent. They are just two dichotomies of motivation from two different psychological perspectives of different research purposes. Due to some overlapping factors, most cases of intrinsic motivation are within the scope of integration, while most of extrinsic motivation proves to be useful.

\section{Motivation Theory of Psychology}

The exploration of motivation is actually to seek answers to the basic questions of why human beings are like them. In the past 100 years, psychologists and scholars have come up with various answers from different perspectives. In the following comments, we discuss some of the leading and influential theories chosen in the field of mainstream psychology: behavioral theory, humanistic theory, cognitive theory and socio-cultural theory.

\subsection{Behavioral Theory}

The early research of behaviorism was based on the investigation of animal behavior. Researchers found that the nature and arrangement of reward system is the most effective way to motivate expected behavior. According to this view, motivation plays an important role on learners, mainly with the help of external 
forces, such as reward or punishment. When reward or punishment takes effect on learners, and then through reinforcement, "expected behavior" will gradually become a habit, good or bad. It is believed that teachers can adopt any form of reinforcement principle, reward or punishment to enliven the classroom atmosphere of students after careful analysis of their real situation. Although behaviorism clarifies some human behaviors, it overemphasizes the use of external force.

Behaviorist psychologists focus on the connection between stimulus and behavior, and emphasize the study of explicit behavior. Watson first put forward behaviorism psychology, but he thought that the purpose of psychology was to describe, explain and control behavior. Therefore, classical behaviorism tended to regard people's psychological process, and lacked interest in explaining the internal causes of behavior. In addition, behaviorism overemphasizes the decisive role of environmental stimulation in human behavior, which naturally denies the role of motivation in human behavior. Therefore, it is easy to understand the reasons behind human behavior too simply and fall into the pattern of mechanical determinism. In the process of explaining people's behavior, people's role is ignored, which inevitably leads to the failure of interpretation. New behaviorism abandons the simplistic and linear explanation of classical behaviorism, and introduces the concept of intermediary variable. The explanation idea also changes from "stimulation behavior" to "stimulation intermediary variable behavior". The development of new behaviorism represents the return of subjective consciousness in psychology, which establishes the subjective status of consciousness in psychological research. Behaviorism has always been favored by educators because it emphasizes the postnatal environment and the role of reward and punishment.

\subsection{Humanistic Theory}

Although behavioral theory emphasizes the importance of external forces, humanistic theory goes deep into internal forces. In this category, motivation is interpreted as the self realization of human potential or needs. As a popular and influential incentive theory with systematic level, Maslow's demand level is the representative of humanistic theory (Maslow, 1987). He listed five different levels: physiology, assurance and security, sense of belonging and love, respect and self actualization, starting from biological needs and developing upward to psychology. People have the motivation to meet the lowest level of demand, and then seek to meet the highest demand. Learning from the level, teachers can encourage students to develop their ability, self-esteem and sense of self realization, and create a positive and free classroom atmosphere to encourage them to learn by establishing a caring and friendly relationship with them. Humanistic psychology has created a new situation for motivation research. In the final analysis, humanistic psychology emphasizes people's free will. Only by recognizing people's independent choice of behavior can motivation research have its foun- 
dation and premise. Maslow, the father of humanistic psychology, put forward the famous "hierarchy of needs theory", which put forward that the highest level of human needs is the need of self realization, laid the foundation of humanistic psychology, and also marked the formation of the theoretical system of humanistic psychology. Maslow emphasizes that need is the basis of motivation, needs reflect the potential significance and value of objects to people, and motivation is the behavior trend based on needs. Maslow believes that there needs to be an instinctive nature. "If we want to protect the weak and micro blog's instinctive needs from being engulfed by a stronger and more favorable culture, we should protect them, not on the contrary." Instinctive needs stubbornly insist on being satisfied, and once frustrated, there will be serious pathological consequences. instinctive needs can only be expressed under appropriate social conditions, which reflects the following characteristics, the difference between man and animal. On the basis of a systematic explanation of human motivation, Maslow emphasizes that human beings have the instinct of transcending animals and puts forward the need of self realization. Humanism emphasizes people's self-development and growth. The enlightenment for educators is to regard students as the main body of learning rather than the container. One of the fundamental tasks of education is to stimulate students' internal self-development and self-improvement. From "what I learn" to "I want to learn", which embodies the ideological connotation of humanistic motivation theory.

\subsection{Cognitive Theory}

Cognitive school regards people's psychological function as information processing system. Cognitive psychology attaches importance to the study of psychological internal process, and takes changing clients' cognition of maladjustment as its fundamental goal. It holds that cognitive distortion is the fundamental cause of bad mood and maladjustment. Once cognitive distortion is changed or corrected, emotional and behavioral disorders will be improved accordingly. The theoretical basis of cognitive model is Beck's cognitive theory of emotional disorders. He believes that "psychological problems" are not necessarily caused by mysterious and irresistible forces. On the contrary, they can arise from ordinary events, such as wrong learning, making wrong inferences based on one-sided or incorrect information, and failing to properly distinguish the difference between reality and ideal, etc. Cognitive process is the information processing process of individual cognitive activities. Cognitive psychology regards cognitive process as a system of information processing according to a certain procedure, which consists of a series of continuous cognitive operation stages, such as information acquisition, coding, storage, extraction and use. The acquisition of information is to receive the stimulating information that directly affects the senses. The function of feeling is to get information. Information coding is to transform one form of information into another, which is beneficial to the storage, extraction and use of information. Individuals have correspond- 
ing information coding methods in perception, representation, imagination, memory, thinking and other cognitive activities. The storage of information is the maintenance of information in the brain. In memory activities, the storage of information has many forms. Information extraction is based on certain clues to find the required information from memory and take it out. The use of information is to use the extracted information for cognitive processing of new information. In the process of cognition, through the coding of information, the characteristics of external objects can be transformed into information in the form of specific image, semantics or proposition, and then stored in the brain. These concrete images, semantics and propositions are actually the manifestation of the characteristics of external objects in individual psychology, and the reflection of objective reality in the brain. Cognitive psychology refers to these specific images, semantics or propositions that reflect the characteristics of objective things in the brain as the psychological representation of external objects, or representation for short. Usually, "Representation" also refers to the representation of external objects in a certain form as the information processing process in the brain. Everyone's emotion and behavior are largely determined by his own way of understanding the world and dealing with the world, that is to say, a person's thought determines his inner experience and reaction. With the latest popular motivational approach, cognitive psychologists focus on cognitive processes that may affect human behavior, such as personal beliefs, expectations and thoughts. It is initiated and regulated by plan, goal, pattern, expectation and attribution. In theories that are closely related to motivation, a cognitive motivation perspective revolves around individuals making decisions about their own actions. In order to make an appropriate decision, it is necessary to know the goal set, the influencing factors, the amount of effort people are prepared to spend in trying to achieve the goal and the possible results. All the factors in the cognitive process of human brain may be directly related to human behavior. In the classroom environment, students can be motivated by teachers to engage in specific work and perform well, not only because of rewards, but also because of incentive factors, such as encouragement to make decisions and ultimately solve problems. Students with different cognitive styles have certain differences in learning styles, subject interests and adaptability to teachers' teaching methods, which will have an impact on students' academic performance. It should be pointed out that there is no absolute distinction between good and bad cognitive style, any cognitive style has its advantages and disadvantages, as long as we can teach according to the characteristics of different cognitive styles, students with any cognitive style can achieve good results.

\subsection{Socio-Cultural Theory}

Vygotsky, the founder of social and cultural theory, talks about the motivation as follows: every function in the development of children's culture appears twice: first, at the social level, and then at the individual level. The core components of 
socio-cultural theory include mediation, internalization, zone of proximal development, scaffolding and activity theory. In the abstract symbolic world, we use language as a psychological tool to mediate transformation, and carry out psychological activities and psychological control. In other words, language symbols play a media role in social and cultural exchanges and interaction, and also affect human thinking activities, which are spontaneous and enable people to make plans and think rationally. Regulation is the main form of mediation. People promote all kinds of human activities by regulating our material world, society and psychology. In foreign language and second language learning, the regulation of others refers to students' cooperative learning with the help of teachers and parents, while self-regulation refers to students' autonomous language learning by relying on their own ability. The same applies to voluntary attention, logical memory and concept formation. All higher functions originate from the actual relationship between human individuals (Vygotsky, 1986). This means that knowledge is the best construction when learners work together. Therefore, cooperation is very important for successful group learning. Learners support each other and encourage the formation, construction and reflection of new materials. Learning is based on real situations and allows groups to reflect on how they can apply knowledge in everyday situations. Social and cultural theory also has good educational application. In the classroom, as a community, teachers plan activities such as group projects, text analysis and peer evaluation to provide students with the opportunity to work with each other to build a solid understanding of the material as it relates to the real world.

The above classification and motivation of psychological exploration are conducive to a better understanding of motivation and human behavior from different perspectives. They recognize the impact of external forces on human beings, go deep into the inner world, and examine the connections between learners and their surroundings. Therefore, they pave the way for future research.

\section{The Enlightenment of Motivation Theory to Teachers' Teaching}

Control motivation level. Every activity has an optimal level of motivation. The middle level of motivation arousal is most conducive to the improvement of learning effect. The middle level motivation makes people's learning efficiency the highest. Lack of motivation or strong motivation will lead to the decline of learning efficiency. Teachers first predict the difficulty of the learning task, and then control the degree of motivation according to the difficulty of the task. When assigning simple tasks, we should increase the pressure of assessment, so that students value the task, in order to achieve better results; if it is a complex task, let students complete the task happily in a relaxed environment. When students encounter difficulties, teachers should give guidance and follow-up, so as to avoid students giving up tasks because of difficulties. But each student's 
level is uneven, the difficulty of the task is different for different students, teachers can divide group appropriately, teach students in accordance with their aptitude. Timely feedback on the completion of learning objectives is conducive to stimulate learning motivation. Feedback promotes the performance of future behaviors with motivational value. Praise students, and help them put success down to their own efforts. If you tell the students how far they are from the goal, they will make more efforts or try other learning methods. If you tell the students that they have reached the goal, they will get a sense of achievement and set a higher goal. What's more, It can help them quickly return to the problem situation and improve their learning efficiency.

\section{Conclusion}

In an era of information explosion society, everyone needs to learn more and constantly update their knowledge. Learner autonomy, as a capability of independent learning, should be given enough attention by English teachers and learners. As English teachers, we should not only know the importance of learner autonomy, but also remember that there are many factors which will influence learner autonomy, including knowing different motivational theory as supplementary to change teaching methods. If we use a motivational way to start from these factors and attach great importance to these factors, we are sure to be of help in promoting the students' learner autonomy and teaching efficiency.

\section{Significance}

It is without doubt that students with strong learning motivation have better English learning effect. It is undeniable that learning motivation is diverse and complex. Stimulating students' learning motivation is a long-term work. Teachers should use the theory to guide the teaching practice, which is believed to help more learners find the correct attribution, stimulate learners' interest in learning, and improve learners' instrumental motivation and integration motivation.

\section{Conflicts of Interest}

The author declares no conflicts of interest regarding the publication of this paper.

\section{References}

Brown, H. D. (1994). Principles of Language Learning and Teaching. Englewood Cliffs, NJ: Prentice Hall.

Deci, E. L. (1975). Intrinsic Motivation. New York: Plenum Near. https://doi.org/10.1007/978-1-4613-4446-9

Dornyei, Z. (2001). Motivational Strategies in the Language Classroom. Cambridge: Cambridge University Press. https://doi.org/10.1017/CBO9780511667343

Maslow, A. H. (1987). Motivation and Personality (3rd ed.). Boston: Addison-Wesley.

Vygotsky, L. (1986). Thought and Language. Oxford: Blackwell. 